Check for updates

Cite this: RSC Adv., 2019, 9, 9820

Received 4th January 2019

Accepted 13th March 2019

DOI: $10.1039 / c 9 r a 00069 k$

rsc.li/rsc-advances

\section{Tunable thermal, mechanical, and controlled- release properties of epoxy phenolic novolac resin microcapsules mediated by diamine crosslinkers}

\author{
Daxia Zhang, (D) $\dagger^{\mathrm{ab}}$ Xianpeng Zhang, (D) $\uparrow^{\mathrm{c}}$ Tongfang Jing, ${ }^{\mathrm{a}}$ Haichao Cao, ${ }^{\mathrm{a}}$ Beixing $\mathrm{Li}^{\mathrm{a}}$ \\ and Feng Liu (iD)*a
}

Diverse shell structures can endow microcapsules (MCs) with a variety of properties. In this study, four kinds of MCs encapsulated with epoxy phenolic novolac resin (EPN) were obtained using diamines as crosslinkers. The FTIR and CLSM confirmed that the EPN-shell was successfully synthesized. SEM results suggested that all four of the MCs possessed smooth and imporous surfaces, and they displayed uniform size distribution, with the average size ranging from 11.2 to $14.8 \mu \mathrm{m}$. Specifically, the MCs synthesized with the crosslinkers of hexamethylene diamine (HAD) and isophorone diamine (IPDA) were more stable and could maintain their spherical shapes in a dry environment, while those synthesized with ethylene diamine (EDA) and triethylene tetramine (TETA) exhibited poorer thermal stabilities and faster release profiles. Lipophilic diamines can form thicker shell thickness, and have smaller $d$-space values and higher Young's modulus values. Therefore, the lipophilicity of the diamine agents is the key factor that influences the specific properties of the MCs. The hybrid approach was validated as a flexible strategy to meet diverse requirements. The present study can provide a comprehensive understanding of diamine crosslinkers in the synthesis of EPNMCs, and such MCs can be extended to a wide range of fields because of their broad tunable performance.

\section{Introduction}

Polymeric microcapsules (MCs) have received growing interest worldwide due to their tunable physicochemical properties and attractive applications and have been widely utilized as drug delivery carriers, microreactors, and encapsulated vectors. ${ }^{\mathbf{1 - 4}}$ When applied in specific applications, smart MCs that can meet controllable demands are highly desired. ${ }^{5,6}$ Furthermore, the microstructure of the capsule shell is the key element that determines the physicochemical properties of the MCs and can be managed by varying the wall-forming materials and operating parameters. $^{7-10}$ For example, MCs fabricated with different polymeric shell materials not only influence the characteristics of their payload cargoes but also affect the UV-resistance ability, permeability, thermal stability and bioactivity of MCs. ${ }^{11,12}$ In addition, some work has also shown that the soft segment length, as well as crosslinker type, can bring obvious diversity to the particle size distribution, morphology, loading content and

\footnotetext{
${ }^{a}$ Key Laboratory of Pesticide Toxicology and Application Technique, College of Plant Protection, Shandong Agricultural University, Tai'an, Shandong 271018, P. R. China. E-mail: fliu@sdau.edu.cn; Tel: +86-0538-8242611

${ }^{b}$ Research Center of Pesticide Environmental Toxicology, Shandong Agricultural University, Tai'an, Shandong 271018, P. R. China

'Laboratory of Medicinal Biophysical Chemistry, Huazhong Agricultural University, Wuhan, Hubei 430070, P. R. China

$\dagger$ D. Z. and X. Z. share joint first authorship.
}

the release behavior of MCs. ${ }^{13-15}$ Therefore, to design and apply microencapsulation techniques more precisely, it is necessary to investigate the effect of coating materials on the structures and properties of the synthesized capsule shell.

Epoxy resins are one of the most versatile classes of polymeric materials that contain two or more epoxy groups in one molecular. ${ }^{16}$ Currently, epoxy resins play an indispensable role in various fields, such as coatings, aerospace, adhesives, paints, and engineering plastics. ${ }^{17-19} \mathrm{Up}$ to now, there were few literatures relevant to the fabrication of microcapsules/microspheres using epoxy resins as shells. ${ }^{20-22}$ Recently, we synthesized MCs with porous surface using epoxy resin and demonstrated that epoxy resin based MCs prepared with a high epoxy value tended to yield a higher crosslinking degree, less porosity and a better thermal stability. ${ }^{22}$ Epoxy resins are reactive monomers and can chemically react with numerous crosslinkers (including imidazoles, diamines, carboxylic acids and polyamides) to form polymeric epoxy systems with a wide range of physicochemical properties, such as excellent chemical resistance and superb mechanical properties. ${ }^{16,23-25}$ Among these crosslinker families, diamines are the most widely used agents owing to their wide availability, low cost and fast reaction. Previous work has shown that when aromatic polyamine was used as crosslinking agent, reaction temperature could control the pore size and porosity of the MCs and thus regulate the release of the core materials. ${ }^{26}$ Nonetheless, to the best of our knowledge, the influence of the diamine structure on the microstructure of the MCs has not been comprehensively investigated to date. 
In the present study, four types of MCs were prepared using epoxy phenolic novolac resin (EPN) as wall-forming material, and four diamines were used as crosslinkers, including ethylene diamine (EDA), hexamethylene diamine (HAD), isophorone diamine (IPDA) and triethylene tetramine (TETA), owing to their high reactivity, low viscosity, wide availability and low cost. The oil-soluble $N$-(1-ethylpropyl)-3,4-dimethyl-2,6-dinitrobenzenamine (Nd) was chosen as a model payload due to its autofluorescence. The surface morphology, particle size, entrapment efficiency and mechanical property of the as-synthesized MCs, as well as the crosslinking degree and thickness of the shells, were characterized elaborately. This study would provide insight on the properties of MCs, which are affected by the structure of the diamine crosslinkers. Additionally, these findings can be an important reference for the precise design and development of MC system.

\section{Materials and methods}

\subsection{Materials}

Epoxy phenolic novolac resin (EPN) was obtained from the Shanghai Resin Plant, China. The resin has an epoxy functionality of $0.44 \mathrm{~mol}$ per $100 \mathrm{~g}$ of resin. Ethylene diamine (EDA, purity $\geq 96.5 \%$ ), hexamethylene diamine (HAD, purity $98 \%$ ), isophorone diamine (IPDA, purity $\geq 99 \%$ ) and triethylene tetramine (TETA, purity $\geq 98 \%$ ) were purchased from SigmaAldrich Chemical Co. $N$-(1-Ethylpropyl)-3,4-dimethyl-2,6dinitrobenzenamine ( $\mathrm{Nd}$, purity 99\%) was purchased from Sigma-Aldrich Chemical Co. Polyoxyethylene sorbitan monooleate (Tween-80) was used as an emulsifier and purchased from Sigma-Aldrich Chemical Co. All reagents were of analytical grade and used without further purification. Deionized water was used throughout all the experiments. Fig. 1 shows the chemical structures of main materials used.

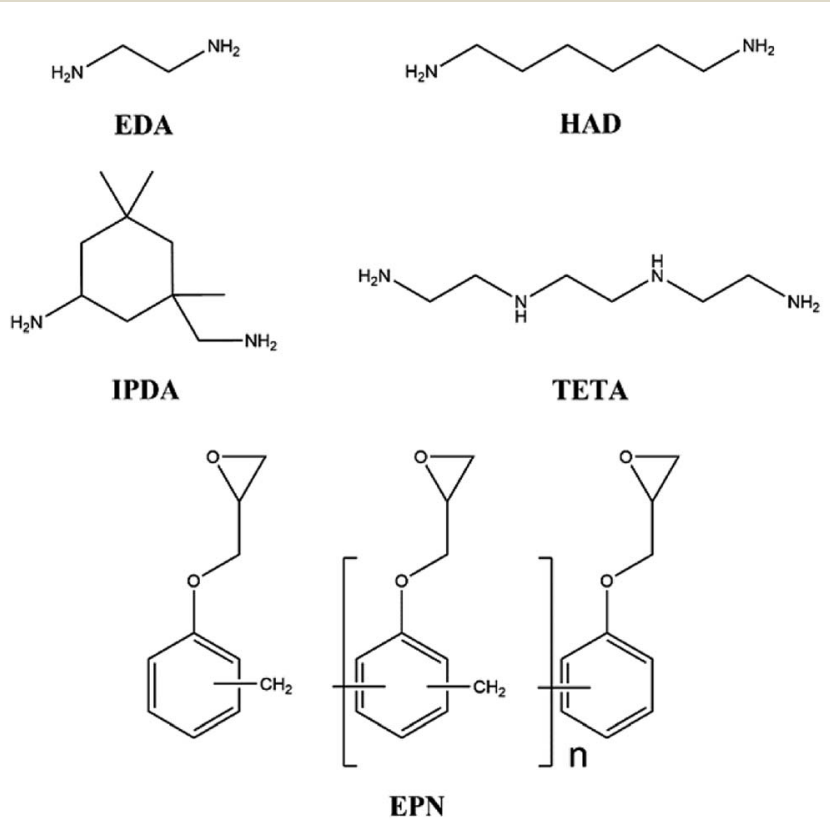

Fig. 1 Chemical structures of the reactive monomers and shellforming material.

\subsection{Preparation of MCs}

The Nd-loaded EDA-MCs were prepared via an interfacial emulsion polymerization technique following a previous publication. ${ }^{26}$ Briefly, Nd and EPN were dissolved in xylene by vigorous agitation to produce a uniform oil phase, while Tween80 (1.5 wt $\%)$ was dissolved in deionized water to obtain the aqueous phase. The oil phase was mixed with the aqueous phase and homogenized at $8000 \mathrm{rpm}$ for $100 \mathrm{~s}$ at room temperature to form a stable oil-in-water $(\mathrm{O} / \mathrm{W})$ emulsion. Then, the emulsion was placed in a three-neck round-bottom flask equipped with a water thermostat bath and mechanical stirrer. Subsequently, an EDA solution (5 wt \%) was added dropwise into the emulsion at a stirring rate of $300 \mathrm{rpm}$. The system was heated to $75{ }^{\circ} \mathrm{C}$ and allowed to react for $5 \mathrm{~h}$ with continuous stirring at $300 \mathrm{rpm}$. MC particles were separated by centrifugation and washed three times with deionized water and then dried in a vacuum oven at $40{ }^{\circ} \mathrm{C}$ for $72 \mathrm{~h}$. The preparation procedures of HAD-MCs, TETA-MCs and IPDA-MCs were similar to that of EDA-MCs, except for changing the type of diamine.

\subsection{Characterization of MCs}

The surface morphologies of the four types of MCs and MC shells in water and dry were observed on a scanning electron microscopy (SEM, Hitachi S4800, Tokyo, Japan) at an accelerating voltage of $3 \mathrm{kV}$ and confocal laser scanning microscopy (CLSM, LSM880 Airyscan, Zeiss, Germany), respectively.

The particle sizes and size distributions of the MCs were measured by a laser particle size analyzer (Mastersizer 2000, Malvern, UK).

The chemical structures were analyzed using a Fourier transform infrared (FTIR) spectrometer (Nicolet 6700, Madison, Wisconsin).

Thermal stabilities of the MCs were determined by a SDTQ600 thermogravimetric analysis (TGA, TA Instruments-Waters LLC, USA) at a heating rate of $10^{\circ} \mathrm{C} \mathrm{min}^{-1}$ from 25 to $700{ }^{\circ} \mathrm{C}$.

The crystallinities of the MC shells were performed on an $\mathrm{X}$ ray diffractometer (XRD, Discover 8, Bruker, Germany).

The mechanical properties of the single $\mathrm{MC}$ was determined using an atomic force microscopy (AFM) with a ScanAsyst-air probe (Bruker, Germany) at $25{ }^{\circ} \mathrm{C}$.

The contact angles of the diamine solutions were determined using a static optical contact angle goniometer (JC2000C2, Shanghai Zhongchen Digital Technology Apparatus Co., Ltd., China). ${ }^{27}$ Briefly, one droplet of the diamine solution was slowly deposited on glass slide and photographed to analyze the values by the cross method.

\subsection{Entrapment efficiency and drug loading content of MCs}

The measurement for the entrapment efficiencies of the MCs was following a method by adapting Yang et al. ${ }^{28} \mathrm{MC}$ suspensions were accurately weighed and then transferred to a glass bottle. Subsequently, hexane $(50 \mathrm{~mL})$ was added and placed the bottle onto a rotary shaker at $70 \mathrm{rpm}$ for $15 \mathrm{~min}$ to dissolve the $\mathrm{Nd}$ that was outside the MCs. After that, the upper hexane layer, which contained the unencapsulated $\mathrm{Nd}$, was measured by 
a high-performance liquid chromatography (HPLC). The total amount of Nd in MC suspensions was determined after the MCs were disrupted with ultrasound.

The Nd loading contents were measured by disruption of the MCs. ${ }^{29}$ Briefly, the dried MC samples were accurately weighed and dissolved in methanol $(100 \mathrm{~mL})$ and then sonicated for 60 min to break the MCs. Subsequently, the mixture was centrifuged and filtered to produce a clear solution for HPLC analysis. The HPLC system (Agilent 1200; Agilent Technologies; Santa Clara, CA) was equipped with an ultraviolet detector. The chromatographic separation was carried out with a Diamonsil C18 column $(250 \mathrm{~mm} \times 4.6 \mathrm{~mm}$ i.d., $5 \mathrm{~mm})$. The mobile phase was mixed with methanol/water $(90: 10, \mathrm{v} / \mathrm{v})$ at a flow rate of 1 $\mathrm{mL} \min ^{-1}$, and the column temperature was maintained at $30^{\circ} \mathrm{C}$. The injection volume was $20 \mu \mathrm{L}$, and the UV detector was set at a wavelength of $238 \mathrm{~nm}$. The entrapment efficiency and drug loading content were calculated using the following equation: entrapment efficiency $(\%)=\left(N_{0}-N_{1}\right) / N_{0} \times 100$, where $N_{0}$ and $N_{1}$ are the total content of $\mathrm{Nd}$ in the MC suspension and hexane layer, respectively. The drug loading content $(\%)=M_{0} / M_{1} \times 100$, where $M_{0}$ and $M_{1}$ are the total content of $\mathrm{Nd}$ in the methanol solution and dried MC sample, respectively.

\subsection{Release behavior of MCs}

The release behavior of the four as-prepared MCs was investigated by adapting a previously reported method. ${ }^{30}$ The mixture of hexane-ethyl alcohol solvents (160:20, v/v) was used as release medium owing to their excellent dissolving capacity for lipophilic Nd and shortening the trial period. Briefly, the dried MC samples were accurately weighed and added in $100 \mathrm{~mL}$ of hexane-ethyl alcohol solvents and then transferred to a rotary shaker at $70 \mathrm{rpm}$ and $30^{\circ} \mathrm{C}$. Subsequently, $0.5 \mathrm{~mL}$ of the liquid was collected at predetermined intervals from the above system and simultaneously added into the same volume of hexaneethyl alcohol solvents. The liquid was filtered with a $0.22 \mu \mathrm{m}$ filter to form a clear solution for HPLC analysis afterward. The cumulative release proportion was calculated using the following equation: cumulative release proportion (\%) $=C_{t} / C_{0}$ $\times 100$, where $C_{0}$ and $C_{t}$ are the content of $\mathrm{Nd}$ in the dried MC samples and in the release medium at time $t$, respectively.

\section{Results and discussion}

\subsection{Preparation and characterization of MCs}

Four types of EPN-MCs were successfully fabricated via the interfacial polymerization technique, and the synthesis schematic of the EDA-MCs was showed in Fig. 2, which was as a representative to illustrate the encapsulating mechanism. The stable Nd oil-in-water emulsion was first obtained using Tween80 as emulsifier at room temperature. Subsequently, the EDA solution was added into the mixture with a stirring rate of $300 \mathrm{rpm}$. With increasing temperature up to $75{ }^{\circ} \mathrm{C}$, the polyaddition reaction between EPN and diamine occurred and the high-crosslinked shells was formed on the surface of oil droplets. This reaction was complex and multiple, and the polymerization mechanism of epoxy groups and diamines were depicted in Fig. 2. Finally, the core-shell MCs emerged with highly cured shells after continued $5 \mathrm{~h}$.

FTIR spectroscopy was conducted to analyze the chemical structure of the MCs. The FTIR spectra of the four samples were similar, as they have the same functional groups. The infrared spectrum of HAD-MCs is shown in Fig. 3 as one of representative examples. The characteristic absorbance peak of epoxy ring at $830 \mathrm{~cm}^{-1}$ disappeared in the cured shell, ${ }^{31}$ indicating that the EPN reacted with the diamine completely. The characteristic peaks of HAD-MCs from 3300 to $800 \mathrm{~cm}^{-1}$ are superpositions of the absorbance peaks of the MC shell at 1604 and $1112 \mathrm{~cm}^{-1}$ ( $\mathrm{C}=\mathrm{C}$ stretching and $\mathrm{C}-\mathrm{O}-\mathrm{C}$ stretching, respectively) and the absorbance peaks of $\mathrm{Nd}$ at 1536 and 1326, 1248 and $1187 \mathrm{~cm}^{-1}$ (nitro groups stretching and $\mathrm{C}-\mathrm{N}$ stretching, respectively). ${ }^{32}$ Additionally, no new peaks were observed in the spectrum of the HAD-MCs. These results indicate that $\mathrm{Nd}$ was successfully encapsulated and no reaction between Nd and EPN occurred.

CLSM was used to observe the morphology and dispersibility of the resulting MCs in water. As shown in Fig. 4, Nd-loaded MCs exhibit intense green fluorescence owing to the autofluorescence of Nd. All four kinds of MCs had spherical shapes and excellent monodispersities without bulk aggregation in water. There were no fluorescent particles observed outside the MCs, suggesting that the vast majority of the payload were successfully encapsulated in the intracapsular cavity.

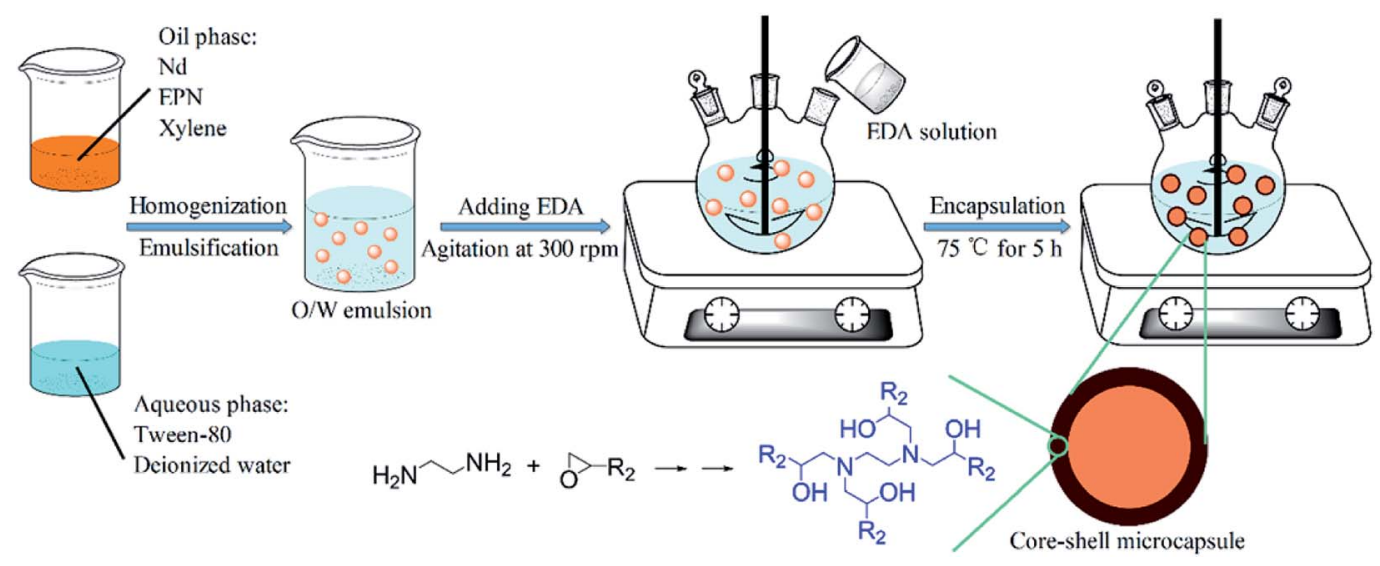

Fig. 2 Schematic illustration of the preparation of EPN-MCs via interfacial polymerization technique. 


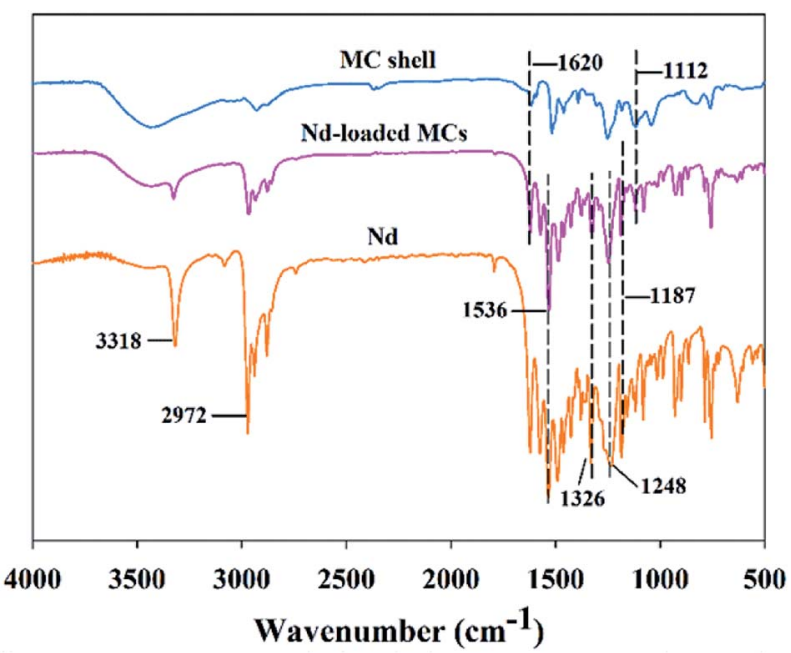

Fig. 3 FTIR spectra of Nd, MC shell and Nd-loaded MCs prepared with $H A D$
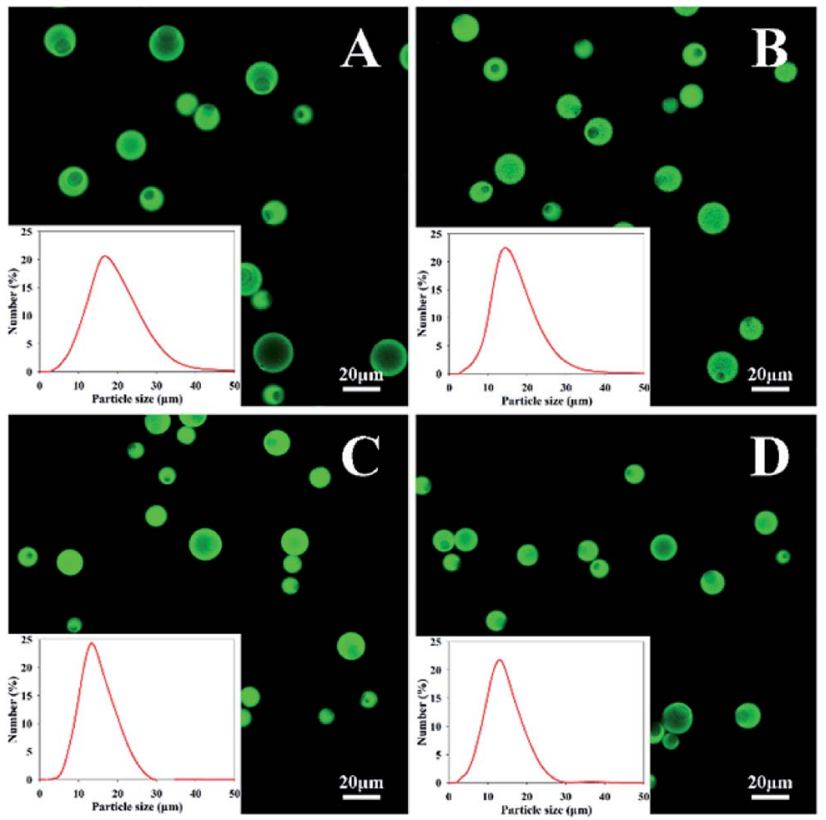

Fig. 4 CLSM images of MCs prepared with (A) EDA, (B) TETA, (C) HAD and (D) IPDA in water. The insets are the particle size distributions of MCs.

Additionally, they also exhibit uniform size distribution with mean sizes in the range of approximately $11.2-14.8 \mu \mathrm{m}$, indicating that diamine structure has no obvious effect on the MC size. This may because that once the diamine monomer was added, the fast polyaddition reaction of wall-forming could trigger and achieve an effective pack for the oil droplets, leading to an integrated confined core-shell structure.

Fig. 5 shows the entrapment efficiencies and loading contents of the four MCs. The encapsulation efficiencies of all samples were above $94 \%$ and they were slightly elevated in the order of EDA-MCs (94.4\%), TETA-MCs (95.2\%), HAD-MCs (96.7\%), and IPDA-MCs (97.8\%), which was in accordance with the CLSM results (Fig. 4), indicating that the oily core was effectively encapsulated in the EPN-shells. Moreover, the loading contents

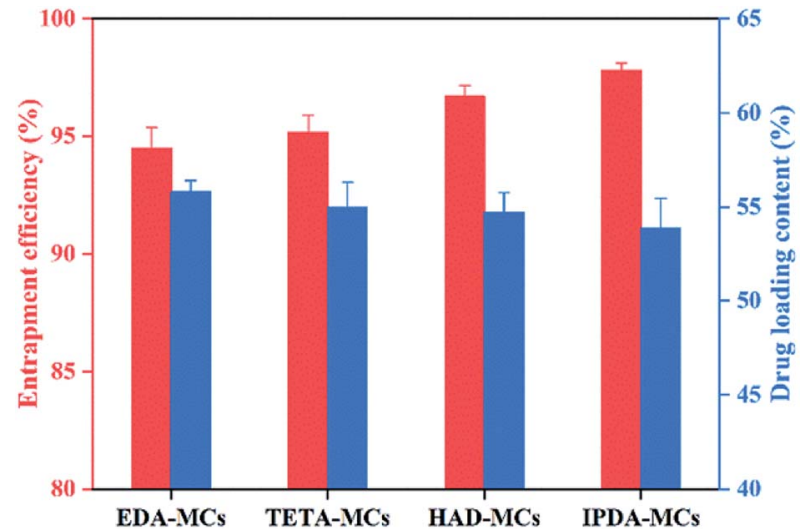

Fig. 5 Entrapment efficiency and the drug loading content of the MCs prepared with four diamine crosslinkers.

were $53.9-55.8 \%$ without considerable variation for the different samples. The cargo-loaded MCs with high entrapment efficiency and drug loading content were highly desired in the practical application. ${ }^{29}$ Based on the above results, it can be concluded that epoxy resins are promising candidates to fabricate core-shell MCs for encapsulating oily cargoes.

\subsection{Morphologies and distributions after drying}

The surface morphologies of as-prepared MCs in a dry state were observed by CLSM and SEM (Fig. 6). These four types of MCs exhibited various morphologies after air-drying (Fig. 6B). The EDA-MCs and TETA-MCs were balloon-like and adhered to each other with bulk agglomeration, some MCs even exhibited wrinkled surface with slight leakage of the loaded drug after air-drying (Fig. 6B1 and B2). CLSM images also showed similar agglomeration for both samples (Fig. 6A1 and A2), which was accordant with the SEM results. Furthermore, EDA-MCs seriously collapsed, with part of the core leaking (Fig. 6C1). Simultaneously, TETA-MCs presented visible wrinkling and collapsing (Fig. 6C2) after prolonged exposed to air, which suggested they burst quickly in dry conditions. In contrast, HAD-MCs and IPDA-MCs were spherically shape that some small dimples existed on their dense and smooth surface (Fig. 6B3 and B4) after air-drying. CLSM images also showed that the MCs separated with each other without agglomeration and showed relatively weak fluorescence intensity (Fig. 6A3 and A4). Moreover, HAD-MCs and IPDA-MCs kept their structural integrity and scarcely encapsulated cargoes escaped from MCs cavity, even after air-drying for $12 \mathrm{~h}$ (Fig. 6C3 and C4). Therefore, the diamines can bring a variety of widely morphologies and shape change to the as-synthesized MCs. The various appearances of the MCs are due to the change in shell structure. Afterwards, the structural characteristics of the capsule shells and the mechanism influenced by the diamine structure was studied.

\subsection{Thermal stabilities and release properties}

The thermal stabilities of the as-prepared MC samples were determined by TGA, as shown in Fig. 7A. The weight loss before $130{ }^{\circ} \mathrm{C}$ is probably due to the evaporation of water adsorbed in the MCs, and that of between 130 and $280{ }^{\circ} \mathrm{C}$ can be ascribed to the decomposition and evaporation of solvent and $\mathrm{Nd}^{33}$ The 

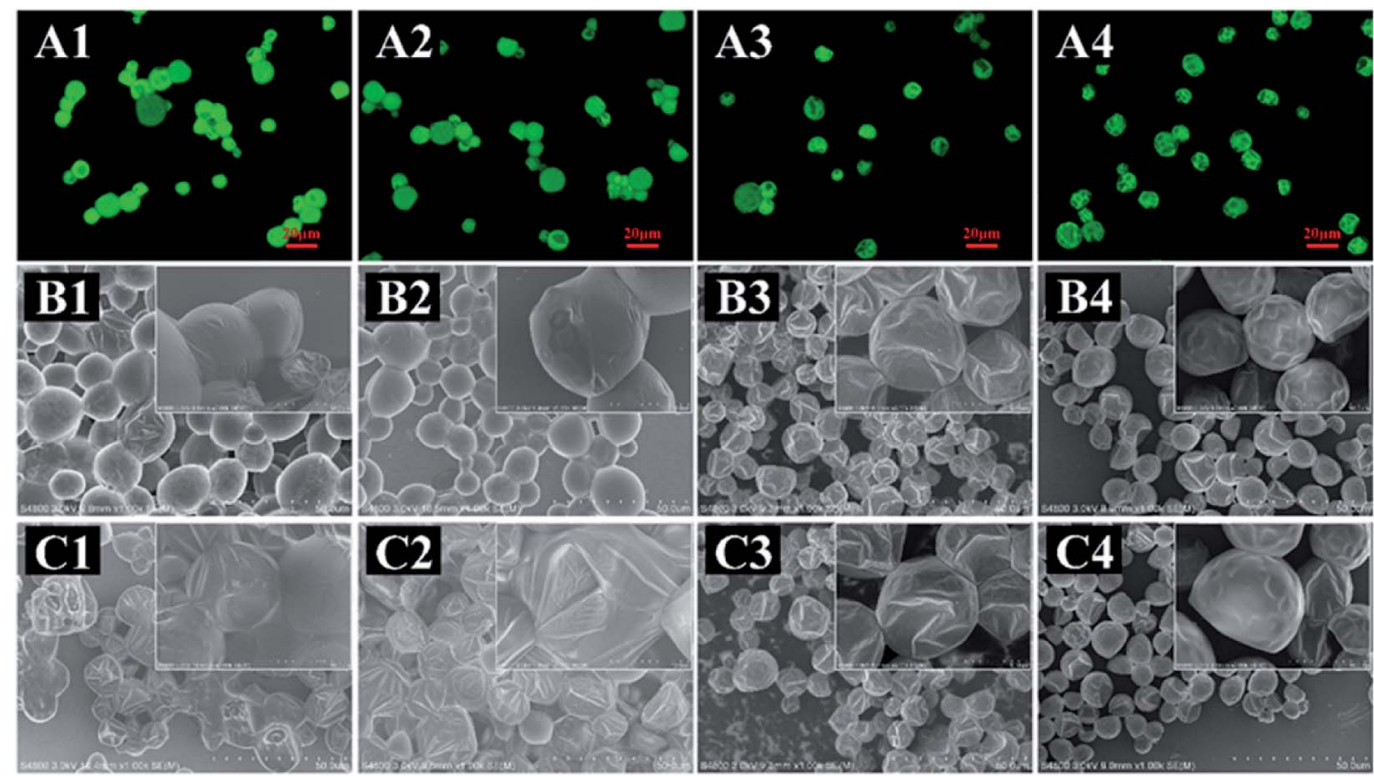

Fig. 6 CLSM images (A: air-dried) and SEM images (B: air-dried; C: air-dried 12 h) of MCs prepared with (1) EDA, (2) TETA, (3) HAD and (4) IPDA.

decomposition of EPN shells started approximately $280{ }^{\circ} \mathrm{C} . .^{22}$ Comparably, the onset temperatures in which the encapsulated cargos began rapidly escaping from the EDA-MCs, TETA-MCs, HAD-MCs and IPDA-MCs were 152, 178, 213 and $220^{\circ} \mathrm{C}$, respectively, indicating that MCs prepared with HAD or IPDA as crosslinkers possessed superior thermal stabilities. Moreover, the release behaviors of the obtained MCs were investigated using a mixed release medium of hexane and ethyl alcohol at room temperature, as shown in Fig. 7B. The cumulative release rates of Nd-loaded MCs ranked as EDA-MCs > TETA-MCs > HAD-MCs > IPDA-MCs under the same exposed time. The cumulative release of EDA-MCs and TETA-MCs reached $98.6 \%$ and $92.6 \%$ after $6 \mathrm{~h}$, respectively, while the cumulative release of HAD-MCs and IPDAMCs merely reached $50.8 \%$ and $33.2 \%$ after $10 \mathrm{~h}$, then they increased slowly at a longer period. The controlled release behavior of the MCs is crucial to application, which is highly dependent on the microstructure of the MC shells., ${ }^{5,12}$ In this study, the hexane/ ethyl alcohol mixture was used as a model to characterize the different release profiles of MCs affected by the diamine types. When applied in specific applications, the influences of external stimuli conditions (such as $\mathrm{pH}$, light, temperature) could be included according to the practical environment. The results of this study confirm that the release rate of EPN-MCs is easily tunable by choosing the appropriate diamine agent, which is beneficial to meet different requirements. Previously, we have successfully fabricated EPN-MCs loaded pendimethalin, and demonstrated that such MCs could achieve tunable release behavior and herbicidal activity by adjusting the epoxy value, which indicated the potential application of EPN-MCs in agriculture. ${ }^{22}$ Such polymeric MCs were suitable to encapsulate the hydrophobic cargoes, and thus have broad utility in agriculture and medicine, etc.

\subsection{Contact angles of the diamine solutions}

The above-mentioned results suggested that the MCs prepared with different diamine agents exhibited a quite visible diversity in morphology, thermal stability and release behavior. The structure of four diamines is a key factor in such differences. Fig. 8 shows the contact angles of the four diamine solutions at
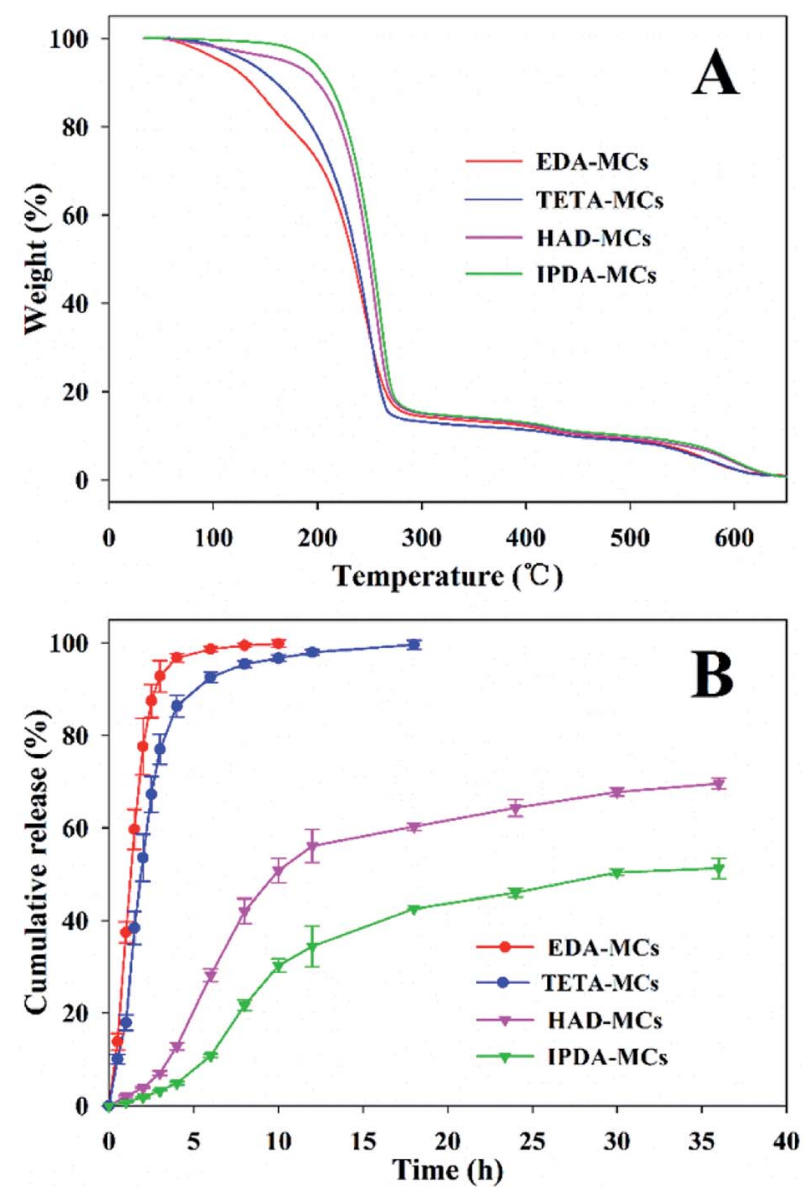

Fig. 7 TGA curves (A) and release properties (B) of EPN-MCs prepared with four diamine crosslinkers. 
a fixed concentration of $5 \mathrm{wt} \%$. When one droplet was slowly deposited on the glass slide, the EDA sample measured a maximal contact angle $\left(42.8^{\circ}\right)$, and the values declined slightly in the order of TETA $\left(40.4^{\circ}\right), \operatorname{HAD}\left(35.5^{\circ}\right)$ and IPDA $\left(30.8^{\circ}\right)$. It can be seen that the interfacial properties of the four diamine solutions are significantly different. The hydrophilic-lipophilic capacity differences of the diamine agents may contribute to the diverse interfacial properties. IPDA possess a hexatomic ring, which leads to the greatest lipophilicity; the remaining three are linear chain diamines, and their lipophilicity was enhanced with an increase in chain length. Laguerre and co-workers demonstrated that alkyl esters with longer chain length resulted in their lower level in the water phase, ${ }^{34}$ which was in accordance with our findings. In this study, the reaction of the diamine with EPN formed a shell via interfacial polymerization, lipophilic diamines are more likely to penetrate the oil droplets through the oil-water interface and react more fully with EPN, which may further affect the crosslinking degree, compactness and thickness of MC shells.

\subsection{Thickness, crystallinity and mechanical property of MC shells}

MC shells obtained by removing the core of the MCs were observed by SEM to characterize their morphology and thickness. As depicted in Fig. 9A, the shells were irregular shapes, owing to the removal of the loaded core. Among them, the EDAMC and the TETA-MC shells displayed a strongly corrugated shrinking shapes, with some weak shell fragments (Fig. 9A1 and A2). The shells became progressively more rigid and undeformed morphologies for HAD-MC and IPDA-MC samples (Fig. 9A3 and A4), suggesting an enhancement of shell rigidity. The fold is formed by stacking two layers of shells. Based on this, the thickness of the capsule shell was calculated. The shell thickness of EDA-MCs was the thinnest, with an average of $65 \mathrm{~nm}$, while the IPDA-MC had thickest shell, which was approximately $158 \mathrm{~nm}$ (Fig. 9D). The TETA-MC and HAD-MC were 84 and $138 \mathrm{~nm}$, respectively. Previous report demonstrated that the thicker MC shell reduced the threat for aquatic

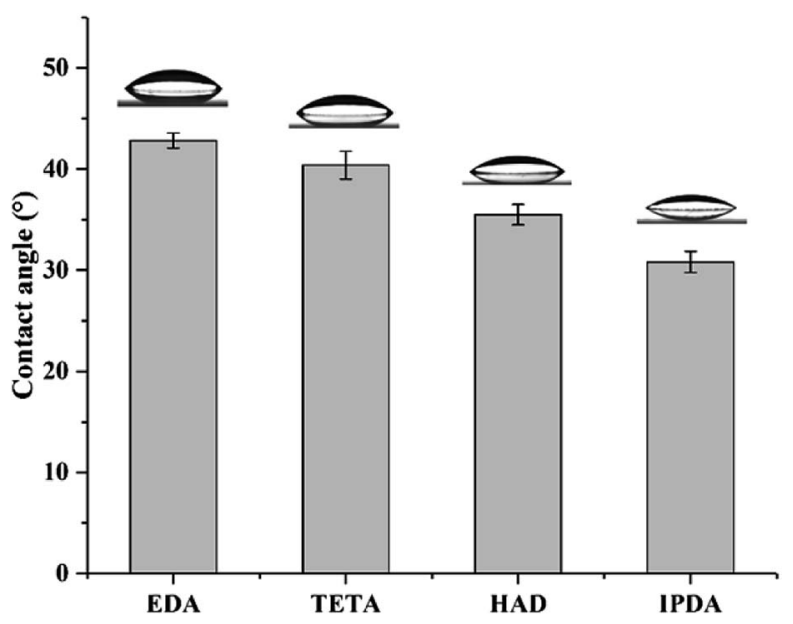

Fig. 8 Contact angle of the four diamine solutions at 5 wt $\%$ fixed concentration on glass surface. organisms due to the poor permeability and leaking of encapsulated core ${ }^{35}$ which is in accordance with our results.

The crystallinity of the cured shells was also examined by XRD. All four shell systems exhibited broad diffraction peaks at a $2 \theta$ angle range of $17.5-21^{\circ}$ (Fig. 9C), indicating that they were amorphous. ${ }^{36}$ Moreover, the most intense peak was the IPDAshell, and the peak intensity declined in the order of HADshell, TETA-shell and EDA-shell. The XRD results revealed the crosslinking degree of shell systems, which was adjusted by the structure of diamine agents. The crosslinking degree of shells fabricated with IPDA or HAD were higher owing to their high lipophilicity, leading to more rigid shell systems. Additionally, calculated from the location of peaks, the average $d$-space of the IPDA-, HAD-, TETA-, and EDA-shell were 3.38, 3.83, 4.37 and $4.53 \AA$, respectively, indicating that the former systems were more densely compacted..$^{37}$

The mechanical properties of the obtained MC shells were also investigated by atomic force microscopy (AFM, Fig. 9B), and the value of Young's modulus for the MC shells were summarized (Fig. 9D). Overall, the MC shells are softer than previously reported polyurethane or urea-formaldehyde MCs, which is shown by their higher modulus. ${ }^{38,39}$ The Young's modulus represents the stiffness of materials and is used to describe their elastic deformation. ${ }^{40}$ In this study, the index is strongly linked to the thickness of MC shell. The average Young's modulus of shell for the EDA-MC, TETA-MC, HAD-MC and IPDA-MC were 7.06, 20.2, 77.3 and 83.8 MPa, respectively. Based on above observations, it can be found that the thickness, crosslinking degree and mechanical strength of the capsule shells are closely correlated with the structures of diamines. The diamine crosslinker contained hexatomic ring (IPDA) or long chain length (HAD) is more hydrophobic, which is more likely to contact with oil phase, further accelerating the polymerization reaction. Therefore, the diamines with higher lipophilicity increased the thickness, stiffness and compactness of the MC shells, and further prevented the core from leaking. In addition, the multiformity of reactivity degree of diamines may produce differences in hydroxyl functionalities on the surface of capsule shells, which can further influence the hydrophilicity/ hydrophobicity of the obtained MCs. In the present work, we only focused on the effects of diamine types on the main structures and controlled release functions of as-prepared microcapsules, the effects of diamine types on hydrophilicity/ hydrophobicity of entire MCs need for further investigated.

\subsection{Combination of different MCs for programmed sequential release}

Given the complexity of practical applications, the MCs with single release behavior probably cannot suit specific environments; and it is a convenient and versatile approach to achieve tunable release property of MCs by mixing various MCs that contain single release characteristics. Based on this, the fastrelease (EDA-MCs or TETA-MCs) and sustained-release MCs (HAD-MCs or IPDA-MCs) were mixed, and their release properties were measured. As shown in Fig. 10, the cumulative release of the hybrid samples that contained EDA-MCs and 



Fig. 9 (A) SEM images and (B) mechanical properties of MCs prepared with (1) EDA, (2) TETA, (3) HAD and (4) IPDA crosslinkers; (C) crystallinity of MC walls; (D) mean thickness and Young's modulus of MCs.

HAD-MCs reached $67.3 \%$ after $10 \mathrm{~h}$, and then the payload sustained slow release for a prolonged exposure duration. The mixture of TETA-MCs and HAD-MCs also displayed similar results, in which the cumulative release reached $56.4 \%$ and $74.5 \%$ after 10 and $35 \mathrm{~h}$, respectively. The release characterizations of the mixed MCs were diverse compared to that of the single MCs. Thus, a tank mix with two or more single releases of MCs will provide more flexibility and a convenient strategy to balance the initial and persistent release. ${ }^{41}$ Even, multiple preparations with different release properties can be mixed to meet the needs of practical applications. Similarly, a previous

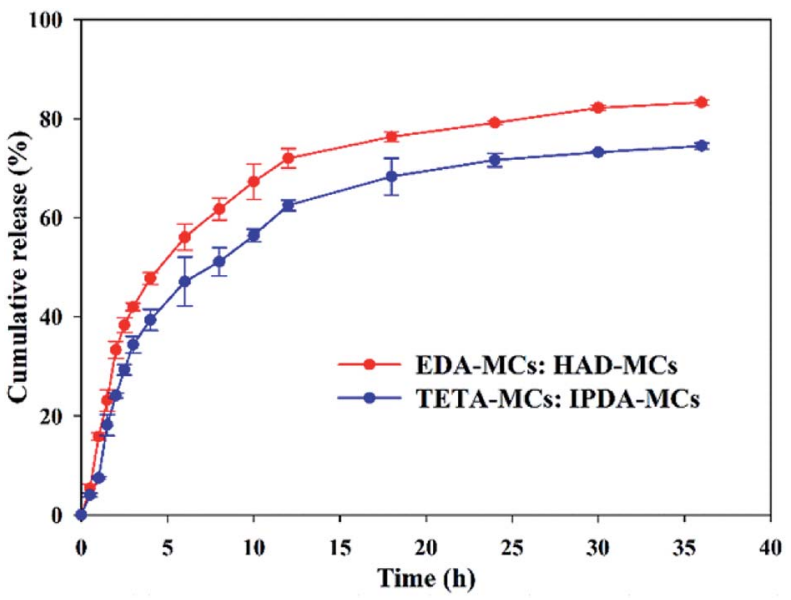

Fig. 10 Controlled release behaviors by hybridizing different MCs at a feed ratio of $4: 6$. study reported that MCs loaded with an oily core containing both free drug molecules and drug-loaded poly(lactic-co-glycolic acid) nanoparticles could achieve programmed sequential drug release. ${ }^{42}$ Our results suggested that the release rate of MCs could be controlled by adjusting diamine type, which was beneficial for controlling payload release to meet different requirements. However, we only chose one feed ratio to verify the hypothesis of hybrid application, and whether they would display new release behavior in other feed ratios or specific conditions, needs to be further examined.

\section{Conclusions}

Four kinds of EPN-MCs with different diamine crosslinkers were prepared through an interfacial emulsion polymerization technique. All four MCs had uniform size distributions, high entrapment efficiencies (more than 90\%) and loading contents (48-52\%). Specifically, high lipophilic diamine agents (HAD and IPDA) enabled to form higher crosslinking degree and thicker of shells, and further led to excellent thermal stabilities, mechanical properties and more sustained release of as-synthesized MCs. Comparably, MCs prepared with poor lipophilic diamine agents (EDA and TETA) were more deformation and burst due to their thin shells and poor mechanical strength. Therefore, the structure of the diamine led to visible differences in the obtained MCs. Based on this, a flexible and versatile strategy was developed to achieve controllable sequential drug release by mixing different types of MCs. This study provides a comprehensive investigation on the physicochemical properties of EPN-MCs prepared with 
different diamines crosslinkers. Such MC systems can be extended to a broad range of other applications in drug delivery, biosensing and phase change materials.

\section{Conflicts of interest}

There are no conflicts to declare.

\section{Acknowledgements}

This work was supported by grants from the National Key R\&D Program of China (2017YFD0200300) and National Natural Science Foundation of China (31772203).

\section{Notes and references}

1 E. Jeoffroy, A. F. Demirörs, P. Schwendimann, S. D. Santos, S. Danzi, A. Hauser, M. N. Partl and A. R. Studart, ACS Appl. Mater. Interfaces, 2017, 9, 37364-37373.

2 B. Li, X. Li, Y. Liu, D. Zhang, J. Lin, W. Mu and F. Liu, Front. Chem., 2018, 6, 1-8.

3 L. Wang, L. Yu, C. Zeng, C. Wang and L. Zhang, Langmuir, 2018, 34, 7106-7116.

4 C. Sui, J. A. Preece, S.-H. Yu and Z. Zhang, RSC Adv., 2018, 8, 29495.

5 A. Abbaspourrad, N. J. Carroll, S.-H. Kim and D. A. Weitz, J. Am. Chem. Soc., 2013, 135, 7744-7750.

6 C. Martino, S.-H. Kim, L. Horsfall, A. Abbaspourrad, S. J. Rosser, J. Cooper and D. A. Weitz, Angew. Chem., 2012, 124, 6522-6526.

7 Q. Ji, C. Guo, X. Yu, C. J. Ochs, J. P. Hill, F. Caruso, H. Nakazawa and K. Ariga, Small, 2012, 8, 1-5.

8 R. Palomba, A. L. Palange, I. F. Rizzuti, M. Ferreira, A. Cervadoro, M. G. Barbato, C. Canale and P. Decuzzi, ACS Nano, 2018, 12, 1433-1444.

9 X. Liu, D. Appelhans, T. Zhang and B. Voit, Macromolecules, 2018, 51, 1011-1019.

10 F. Ran, W. Lei, Y. Cui, J. Jiao, Y. Mao, S. Wang and S. Wang, J. Colloid Interface Sci., 2018, 511, 57-65.

11 D. X. Zhang, B. X. Li, X. P. Zhang, Z. Q. Zhang, W. C. Wang and F. Liu, J. Agric. Food Chem., 2016, 64, 2841-2846.

12 J. Shi, C. Yang, S. Zhang, X. Wang, Z. Jiang, W. Zhang, X. Song, Q. Ai and C. Tian, ACS Appl. Mater. Interfaces, 2013, 5, 9991-9997.

13 H. Zhang and X. Wang, Sol. Energy Mater. Sol. Cells, 2009, 93, 1366-1376.

14 K. Hong and S. Park, React. Funct. Polym., 1999, 42, 193-200.

15 A. Eceiza, M. D. Martin, K. de la Caba, G. Kortaberria, N. Gabilondo, M. A. Corcuera and I. Mondragon, Polym. Eng. Sci., 2010, 48, 297-306.

16 B. Xu, W. Xu, G. Wang, L. Liu and J. Xu, Polym. Adv. Technol., 2018, 1-11.

17 X. Fan, J. T. Miao, Y. Li, Q. Guan, A. Gu and G. Liang, Appl. Surf. Sci., 2018, 447, 315-324.
18 Z. Li, J. Zhang, F. Dufosse and D. Y. Wang, J. Mater. Chem. A, 2018, 1-11.

19 J.-T. Miao, L. Yuan, Q. Guan, G. Liang and A. Gu, Polym Int., 2018, 67, 1194-1202.

20 O. Pascu, R. Garcia-Valls and M. Giamberini, Polym. Int., 2008, 57, 995-1006.

21 W. Wang, L. Yuan, G. Liang, A. Gu and J. Wu, Colloid Polym. Sci., 2010, 288, 719-729.

22 X. P. Zhang, J. Luo, T. F. Jing, D. X. Zhang, B. X. Li and F. Liu, Colloids Surf., B, 2018, 165, 165-171.

23 P. Maksym, M. Tarnacka, A. Dzienia, K. Matuszek, A. Chrobok, K. Kaminski and M. Paluch, Macromolecules, 2017, 50, 3262-3272.

24 A. S. Rahimi, M. R. Ayatollahi and A. R. Torabi, Polym. Test., 2018, 70, 57-66.

25 L. Zarybnicka, R. Bacovska, M. Vecera, J. Snuparek, M. Alberti, J. Rychly and P. Kalenda, J. Appl. Polym. Sci., 2015, 133, 1-9.

26 X. P. Zhang, J. Luo, D. X. Zhang, T. F. Jing, B. X. Li and F. Liu, J. Colloid Interface Sci., 2018, 517, 86-92.

27 X. P. Zhang, T. F. Jing, D. X. Zhang, J. Luo, B. X. Li and F. Liu, Ecotoxicol. Environ. Saf., 2018, 163, 349-355.

28 Y.-H. Yang, X.-Z. Li and S. Zhang, RSC Adv., 2018, 8, 2998029987.

29 B. Liu, Y. Wang, F. Yang, H. Cui and D. Wu, J. Agric. Food Chem., 2018, 66, 6561-6568.

30 B. X. Li, L. Guan, K. Wang, D. X. Zhang, W. C. Wang and F. Liu, J. Appl. Polym. Sci., 2015, 133, 1-8.

31 F. Mohtadizadeh, M. J. Zohuriaan-Mehr, B. S. Hadavand and A. Dehghan, Prog. Org. Coat., 2015, 89, 231-239.

32 G. Rytwo, Y. Gonen, S. Afuta and S. Dultz, Appl. Clay Sci., 2005, 28, 67-77.

33 Y. Liang, M. Guo, C. Fan, H. Dong, G. Ding, W. Zhang, G. Tang, J. Yang, D. Kong and Y. Cao, ACS Sustainable Chem. Eng., 2017, 5, 4802-4810.

34 M. Laguerre, L. J. L. Giraldo, J. Lecomte, M.-C. Figueroaespinoza, B. Barea, J. Weiss, E. A. Decker and P. Villeneuve, J. Agric. Food Chem., 2010, 58, 2869-2876.

35 B. X. Li, W. C. Wang, X. P. Zhang, D. X. Zhang, Y. P. Ren, Y. Gao, W. Mu and F. Liu, Adv. Funct. Mater., 2017, 27, 1-9.

36 H. Liu, D. Chaudhary, S. I. Yusa and M. O. Tadé, Carbohydr. Polym., 2011, 83, 1591-1597.

37 G. Pan, Z. Du, Z. Chen, C. Li, X. Yang and H. Li, Polymer, 2007, 48, 3686-3693.

38 W. Ma, W. Zhang, Y. Zhao and S. Wang, J. Appl. Polym. Sci., 2016, 133, 1-6.

39 N. Tsuda, T. Ohtsubo and M. Fuji, Adv. Powder Technol., 2012, 23, 724-730.

40 C. Campd, T. M. H. Cdsta, A. Dliveira Rids and S. H. Flôres, Food Sci. Technol., 2016, 4, 1-4.

41 J. Luo, T. F. Jing, D. X. Zhang, X. P. Zhang, B. Li and F. Liu, Colloids Surf., B, 2018, 169, 404-410.

42 X.-L. Yang, X.-J. Ju, X.-T. Mu, W. Wang, R. Xie, Z. Liu and L.-Y. Chu, ACS Appl. Mater. Interfaces, 2016, 8, 10524-10534. 